\title{
Cancer diagnostics and treatment: are we ready to implement PPPM?
}

\author{
Dominic M. Desiderio
}

Published online: 11 September 2010

(C) European Association for Predictive, Preventive and Personalised Medicine 2010

The scientific community is always excited whenever a new journal appears in the open scientific literature. Such was the case when the first issue of the EPMA Journal of International Reviews in Predictive, Preventive and Personalized Medicine (PPPM) appeared in 2010.

This present issue focuses on PPPM in Cancer, and it contains a spectrum of topics that covers the range from basic research, via clinical research, to potential implementation. Each review focuses on one or a reasonable combination of the three main topics embodied in the EPMA journal, and highlights the predictive, preventive, and personalized aspects of cancer treatment. It is clear from the scientific literature, in general, and in each one of the reviews in this issue, in particular, that the next quantum leap in clinical science now derives explicitly from one or more of those three features of medicine: accurate prediction, efficacious prevention, and detailed personalized treatment. The objective of this journal and of this issue on cancer is to present state-of-the-art results from researchers from around the world, and to provide timely reviews of cutting-edge basic research, clinical research, and clinical results. The reviews will range from the molecular level (DNA, RNA, proteins, metabolites), the cellular level, clinical research, and clinical practice.

A brief overview follows of each topic that appears in this current issue of PPPM in Cancer. These reviews cover the following areas of research: the "First Do No Harm" concept; the shift from correlative to predictive oncology; selenium and oxidative stress in cancer; BRCAl and $B R C A 2$ gene mutations; a proposal for non-invasive

D. M. Desiderio $(\bowtie)$

University of Tennessee Health Science Center,

Memphis, TN, USA

e-mail: ddesiderio@uthsc.edu proteomics in breast cancer; gene-expression profiling in breast cancer; proteomics and nitroproteomics of pituitary adenomas; epidemiological analysis of gastric adenocarcinomas; endoscopic diagnosis for the early detection of gastrointestinal cancer; fluoropyrimidine toxicity and polymorphisms of the dihydropyrimidine dehydrogenase gene; and circulating DNA and RNA.

1. The connection between the two concepts "First Do No Harm" and "Prediction in Oncology" is discussed [1]. The authors review data on cancer-treatment modalities that could have either cancer-promoting or cancer-inhibiting effects. Those opposite effects would be obscured in a large-scale Phase III clinical study. On the other hand, the judicious use of "a biomarker"- or more probably "a group of biomarkers"- might accurately distinguish and monitor the cancer-promoting versus the cancer-inhibiting aspects of treatment.

2. A paradigm shift in clinical and statistical investigations in cancer research is proposed as the field moves from correlative science to predictive oncology [2]. Appropriate clinical studies are needed to accurately evaluate the clinical utility of prognostic and predictive biomarkers. This review provides a critical assessment of pertinent terms used in the literature, the various types of "biomarkers", and the desired clinical outcome.

3. A significant amount of cancer research indicates that reactive oxygen species (ROS), reactive nitrogen species (RNS), and oxidative stress play key roles; especially in the mitochondria. Selenium is a key element in those redox and in other processes. Because selenium is in the same column of the periodic table as sulfur, it has closely related chemical properties. A review of the role of selenium in the prevention of 
human cancers is thus timely because selenium modulates all growth [3]. The concentration and the chemical form of selenium in cellular structures might prevent or treat a tumor. Selenomethionine and selenocysteine are both incorporated into proteins, and 25 selenoproteins are known. Selenium supplements and the ability of selenium to interact with metals are discussed.

4. The implications of mutations in Founder BRCA1/2 for the early detection and prevention of hereditary breast-ovarian cancer are discussed [4]. A definition of a founder effect is an effect on gene frequency in a population with a small number of original settlers, or "founders", when one or more founders had that gene. This manuscript reviews BRCA1 and BRCA2 gene mutations in Europe.

5. Non-invasive proteomics is suggested as a method to screen for breast cancer and also for treatment [5]. The accurate analysis of a non-invasive breast cancer susceptibility biomarker (e.g., combined -omics/imaging tests) obtained from blood, saliva, or nipple aspirate would be very welcome. This manuscript proposes such a potential approach for breast cancer.

6. The scientific literature in gene-expression profiling in breast cancer was reviewed [6]. This review describes the research to develop a range of biomarkers to accurately describe the various types and stages of breast cancer. For example, the authors note that subtypes might respond differently to chemotherapy. Clearly, the concept of "one biomarker" is insufficient in the field of breast cancer, and an accurate "panel of biomarkers" is needed. In addition, the proteins that participate in the various types of breast cancer must also be identified. The authors do an excellent service to other researchers in this field because they have reviewed a wide range of gene-expression profiling methods; each method uses a different number of genes in each panel. That wide range of the number of genes in each different panel reflects the complexity of the geneexpression profiles that are needed to accurately indicate each cancer type, the individual chemotherapy that is required, and the improving ability to elucidate breast cancer. One of the objectives of that intensive research is to identify patients who would benefit from minimizing any unnecessary therapy.

7. Proteomics methods were used to analyze pituitary adenomas (micro-; macro-) in order to discover mechanisms that could lead to cancer [7]. Differentially expressed proteins (DEP's) were characterized with mass spectrometry. The discovery of new nitroproteins was reviewed. Nitroproteins derive from ROS and RNS, and might play key roles in cancers. Bioinformatics programs were used to locate those
DEP's and nitroproteins in cellular mechanisms. These basic studies could elucidate the mechanisms that contribute to cancer formation.

8. It is always important to analyze data whenever a particular cancer decreases in the world. Gastric adenocarcinomas are such a case in developed countries (declined in the 1970's), and those epidemiological aspects are reviewed [8]. It is very important to note that the aging world population will contribute to an increase in those cancers. The epidemiology, histology, etiology, prognosis, and prevention of gastric cancer are summarized.

9. It is very important to discover either a cancer or a precancerous lesion accurately and early in a cancer's development. The use of endoscopic diagnosis in the early diagnosis of gastrointestinal cancer is reviewed [9]. Endoscopy plays a key role in the reduction of cancer incidence (early detection; eradicate precursors), and via a reduction of mortality (detect/treat a cancer in the early stages). Improvements in endoscopy are needed in the technology, diagnosis of poorly visible nonpolypoid precursors, treatment, and training.

10. The importance of individual clinical responses to chemotherapy is the focus of a review of colorectal cancer patients [10]. Genetic polymorphisms are important for those patients who undergo fluoropyrimidine chemotherapy in terms of inter-individual variations of drug toxicity, tolerability, and efficacy. Polymorphisms of thymidate synthase, orotate phosphoribosyltransferase, dihydropyrimidine dehydrogenase, methylene tetrahydrofolate reductase, and glutathione S-transferase, and interactions among genotypes are discussed.

11. The connection between fluoropyrimidine toxicity and polymorphisms of the dihydropyrimidine dehydrogenase gene are reviewed [11]. Such a study is important because toxicity leads to discontinued treatment. The complex metabolism of 5-flurouracil and any polymorphism of the associated metabolizing enzymes are crucial to understand. That process vividly demonstrated the need for personalized medicine to accurately predict and effectively prevent a cancer.

12. The possibility to use DNA and RNA, which circulate in plasma and serum, to monitor treatment and for prognosis in oncology are reviewed [12]. Different levels were found between primary tumors and metastases. The circulating nucleic acids were tumor cell-derived. The possible roles that CNAPs might play in personalized cancer diagnosis, treatment monitoring, and prognosis are reviewed. The various cancers that are discussed include lung, colorectal, breast, ovarian, prostate, gliomas, nasopharyngeal, and human papilloma viral carcinomas. 
13. Pertinent features of colorectal cancer (CRC) such as risk factors, family history, diet/lifestyle and chronic inflammation are reviewed [13]. Average-risk individuals must be screened for CRC to detect any latent disease. Physical activity, obesity, smoking and diet play roles in CRC. Six chemoprevention agents are discussed. Family history and genetic components, the correlation between adenomas and CRC, and the significance of the adenoma-carcinoma sequence are discussed. Genetic testing and counseling are reviewed. Extensive ulcerative colitis is closely linked with CRC. Testing is reviewed in a comprehensive fashion, and includes imaging and fecal bleeding. The critical distinction between sigmoidoscopy and colonoscopy is clearly delineated.

It is clear that this issue of The EPMA Journal on Cancer covers a wide range of some of the very important and cutting-edge research as well as potential implementations. It is important to mention that each review-article is designed to cover the status quo and the perspectives in each corresponding field/type of cancer. This issue contains only a fraction of the important "PPPM in cancer" research that is underway around the world. Future issues will review the predictive diagnostics, preventive measures and personalized treatment of other cancer types. State-of-the-art methods and instruments, paradigm shifts, quantum leaps forward, and new hypotheses are all underway in cancer research. Those new and exciting experimental data and clinical results will continue to accumulate in the scientific literature, and they will be reviewed in the future issues that will be dedicated to "PPPM in Cancer" each 3rd quarter in a 2-year cycle (thus, the next PPPM in Cancer is in 2012).

\section{References}

1. Characiejus D, Hodzic J, Jacobs JJL. "First do no harm" and the importance of prediction in oncology. EPMA J. 2010. doi:10.1007/s13167-010-0042-1.

2. Simon R. Moving from correlative science to predictive oncology. EPMA J. 2010. doi:10.1007/s13167-010-0040-3.

3. Björnstedt M, Fernandes AP. Selenium in the prevention of human cancers. EPMA J. 2010. doi:10.1007/s13167-010-0033-2.

4. Janavičius R. Founder BRCA1/2 mutations in the Europe: implications for hereditary breast-ovarian cancer prevention and control. EPMA J. 2010. doi:10.1007/s13167-010-0037-y.

5. Debald M, Wolfgarten M, Walgenbach-Brünagel G, Kuhn W, Braun M. Non-invasive proteomics - thinking about personalized breast cancer screening and treatment. EPMA J. 2010. doi:10.1007/s13167-010-0039-9.
6. Mallmann MR, Staratschek-Jox A, Rudlowski C, Braun M, Gaarz A, Wolfgarten $\mathrm{M}$, et al. Prediction and prognosis: impact of gene expression profiling in personalized treatment of breast cancer patients. EPMA J. 2010. doi:10.1007/s13167-010-0044-Z.

7. Zhan X, Desiderio DM. The use of variations in proteomes to predict, prevent, and personalize treatment for clinically nonfunctional pituitary adenomas, EPMA J. 2010. doi:10.1007/s13167010-0028-z.

8. Sjödahl K, Lagergren J. Epidemiological aspects of gastric adenocarcinoma: are predictive diagnostics and targeted preventive measures possible? EPMA J. 2010. doi:10.1007/s13167-0100043-0.

9. Lambert R. Prevention of gastrointestinal cancer by surveillance endoscopy. EPMA J. 2010. doi:10.1007/s13167-010-0023-4.

10. Schwarzenbach H. Predictive diagnostics in colorectal cancer: impact of genetic polymorphisms on individual outcomes and treatment with fluoropyrimidine-based chemotherapy. EPMA J. 2010. doi:10.1007/s13167-010-0022-5.

11. Del Re M, Di Paolo A, van Schaik RH, Bocci G, Simi P, Falcone A, et al. Dihydropyrimidine dehydrogenase polymorphisms and fluoropyrimidine toxicity: ready for routine clinical application within personalized medicine? EPMA J. 2010. doi:10.1007/ s13167-010-0041-2.

12. Gahan PB. Circulating nucleic acids in plasma and serum: diagnosis and prognosis in cancer. EPMA J. 2010. doi:10.1007/ s13167-010-0021-6.

13. Björk J. Strategies for colon cancer prevention. EPMA J. 2010. doi:10.1007/s13167-010-0047-9.

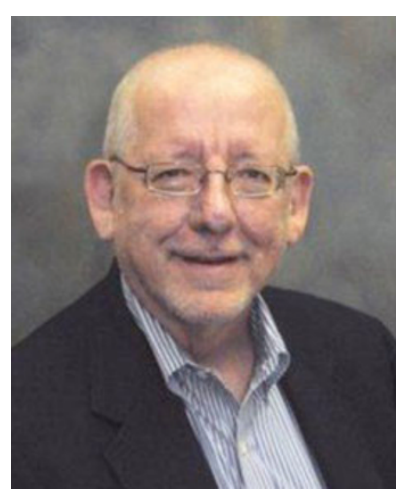

Dominic M. Desiderio received his undergraduate training at the University of Pittsburgh, and his graduate training at the Massachusetts Institute of Technology with Klaus Biemann. After 11 years at the Baylor College of Medicine Institute for Lipid Research, he moved to the University of Tennessee Health Science Center, where he is a Professor of Neurology (Chemistry) in the Department of Neurology, Professor of Molecular Sciences in the Department of Molecular Sciences, and Director of the Charles B. Stout Neuroscience Mass Spectrometry Laboratory. His main area of research focuses on the basic molecular mechanisms that are involved in human pathologies; in particular, pituitary adenomas. He uses proteomics methodologies to analyze differentially expressed proteins, phosphoproteins, and nitroproteins in human tissues and fluids. Other areas of research include the qualitative and quantitative analytical measurements of neuropeptides in tissues and fluids. $\mathrm{He}$ is an editor of Mass Spectrometry Reviews, a co-editor of the Wiley Book-Series on Mass Spectrometry, and Associate Editor of The EPMA Journal. 